2. A. H. Stone, Metrizability of decomposition spaces, Proc. Amer. Math. Soc. vol. 7 (1956) pp. 690-700.

3. A. V. Martin, Decompositions and quasi-compact mappings, Duke Math. J. vol. 21 (1954) pp. 463-469.

4. W. E. Malbon, Invariants for quasi-compact mappings, University of Virginia Dissertation, 1955.

UNIVERSITY OF VIRGINIA

\title{
CONNECTED SETS OF VAN VLECK
}

PAUL M. SWINGLE

Both Vitali and Van Vleck have given interesting constructions of Lebesgue nonmeasurable sets in euclidean $E_{1}$. Here we give a generalization for the construction of Van Vleck for $E_{m}, m \geqq 2$; our interest is in the type of connected set that can be so obtained. Elsewhere we will consider the construction of Vitali. Of interest also is the interlacing of these connected sets.

Below $\Omega$ is the first transfinite ordinal whose cardinal is the same as that of the linear continuum: $\alpha, \beta, \gamma$ are ordinals, $>0$ and $<\Omega$. We will say that the Van Vleck basic set for a given point $P_{\alpha}$ $=\left(x_{1 \alpha}, x_{2 \alpha}, \cdots, x_{m \alpha}\right)$ in $\left(x_{1}, x_{2}, \cdots, x_{m}\right)$-coordinate space is the set of all $\left(x_{1 \alpha}^{\prime}, x_{2 \alpha}^{\prime}, \cdots, x_{m \alpha}^{\prime}\right)$ where for each $j(j=1,2, \cdots, m)$, we have as in $[1$, p. 240],

$$
x_{j \alpha}^{\prime}=\frac{x_{j \alpha}}{2^{( \pm p)}} \pm \frac{u}{2^{v}} \quad(u, v, p=0,1,2, \cdots) .
$$

Van Vleck constructs two complementary sets in $E_{1}$, each a reflection about $x=1 / 2$ of the other; it is in part because measure is invariant under reflection that his construction gives Lebesgue nonmeasurable sets.

We will take the Van Vleck $x_{1}$-reflected set for $P_{\alpha}$ as the set of all $\left(-x_{1 \alpha}^{\prime}, x_{2 \alpha}^{\prime}, \cdots, x_{m \alpha}^{\prime}\right)$ with $x_{j \alpha}^{\prime}$ as above, i.e. it is the Van Vleck basic set for $P_{\alpha}$ reflected about $x_{1}=0$; and the $\left(x_{h}, x_{g}, \cdots, x_{k}\right)$-reflected set for $P_{\alpha}$ is the Van Vleck basic set for $P_{\alpha}$ reflected about the coordinate subspace where $x_{h}=0, x_{g}=0, \cdots, x_{k}=0$, i.e. the set of all $\left(x_{1 \alpha}^{\prime \prime}, x_{2 \alpha}^{\prime \prime}, \cdots, x_{m \alpha}^{\prime \prime}\right)$, where $x_{t \alpha}^{\prime \prime}=-x_{t \alpha}^{\prime}$ for $t=h, g, \cdots, k$ and otherwise $x_{t \alpha}^{\prime}=x_{t \alpha}^{\prime \prime}$ : these reflections include one about the origin, referred to as the $(m-m)$-coordinate space below.

Presented to the Society, November 30, 1957; received by the editors October $10,1957$. 
It is easier to see our desired construction if we state this by means of Zermelo's axiom of choice. Let $M$ be the set of all points of $E_{m}$ which have all coordinates rational: as noted by Van Vleck we must take our points from $E_{m}-M$ in order to have the basic set and its reflections disjoint. Choose then in $E_{m}-M$ a point $P_{1}$. We have dependent upon $P_{1}$ then the Van Vleck basic set for $P_{1}$ together with all the possible $\left(x_{h}, x_{g}, \cdots, x_{k}\right)$-reflected sets for $P_{1}$, the sum of all of which is a countable set which we will call $V_{1}$ and $P_{1} \in V_{1}$. Choose $P_{2} \in E_{m}-M-V_{1}$. Take $V_{2}$ likewise the sum of the Van Vleck basic set for $P_{2}$ and all its reflections. Having for $\alpha<\Omega$ chosen by induction $P_{\beta}$ for all $\beta<\alpha$, choose $P_{\alpha} \in E_{m}-M-\left(V_{1}+V_{2}+\cdots+V_{\beta}+\cdots\right)$, where $V_{3}$ for $P_{B}$ is of similar construction to $V_{1}$ for $P_{1}$.

Let now $S$ be the sum of all the Van Vleck basic sets for every $P_{\alpha}(\alpha=1,2, \cdots, \beta, \cdots ; \beta<\Omega)$ plus the sum of all those $\left(x_{h}, x_{g}, \cdots, x_{k}\right)$-reflected sets for each $P_{\alpha}$ excepting none of $x_{h}, x_{g}, \cdots, x_{k}$ is taken as $x_{m}$. Let $C=E_{m}-(S+M)$. Thus we see that $C$ is the reflection of $S$ about $x_{m}=0$. Also $C$ is the reflection of $S$ about the origin, but that about $x_{m}=0$ is the better for our desired construction of connected sets to give Theorem 1 below. We note now, without repeating the proof, that the methods used by Van Vleck [1, pp. 238-239] will show that $S$ and $C$ are each nonmeasurable Lebesgue; also it is to be noted that these sets can be broken up in such a manner that this can be shown by the same methods used for sets of Vitali; we will not do this here. We note further that with measure invariant under reflection each of the possible $\left(x_{h}, x_{g}, \cdots, x_{k}\right)$ reflected sets of the sum, $W$, of the Van Vleck basic sets for all $P_{\alpha}$ must have the same measure as $W$ has, if $W$ has measure. But there are a finite number of these reflected sets and thus the sum of these for $S$ cannot give the Lebesgue nonmeasurable set $S$, and similarly for $C$. Hence $W$ and each of its $\left(x_{h}, x_{a}, \cdots, x_{k}\right)$-reflected sets must be Lebesgue nonmeasurable.

We wish now to put a condition upon the choice of the $P_{\alpha}$ above which will insure that $S+M$ be connected. We note by Knaster and Kuratowski's well known theorem [2, Theorem 37, p. 233] that $S+M$ is disconnected if and only if there exists a separating boundary continuum $B$ in $E_{m}-(S+M)$, i.e. $C \supset B$. Also because of the homogeneous character [1, p. 238] of Van Vleck's construction of $S$ and $C$ every translation through any distance $\pm u / 2^{v}(u, v=1,2, \cdots)$ in each $x_{j}$ direction must give a translated $B$ contained in $C$. For each $B$ furthermore reflection about $x_{m}=0$ gives a $B^{\prime}$, contained in $S$, which is a separating boundary continuum of $C+M$. Thus, if $B$ is curved, reflection about $x_{m}=0$ will give a $B^{\prime}$ which, when translated, 
will intersect it: this is impossible since $S \supset B^{\prime}, C \supset B$ and $S \cdot C$ is vacuous. Also if $B$ is an $(m-1)$-dimensional generalized plane not of equation $x_{m}=c, c$ a constant, we will get a similar contradiction by the use of the rational translations of $B^{\prime}$. If one $(m-1)$-plane $x_{m}=c$ separates $S$, there are at least a countable infinity of parallel ones which separate it also; and parallel to these are infinitely many that separate $C$. Thus in choosing the $P_{\alpha}(\alpha=1,2, \cdots, \beta, \ldots ; \beta<\Omega)$ by Zermelo's axiom of choice and in putting the Van Vleck basic sets for the $P_{\alpha}$ in $S$ or $C$, if for an $x_{m}=c$ and for $\beta<\alpha$ we have put the Van Vleck basic set for a $P_{\beta}$ in $S$ we must put one for a $P_{\alpha}$ in $C$, i.e. make sure that each $x_{m}=c$ contains points of both $S$ and $C$. Because each basic set is dense in $E_{m}$, it follows that both $S$ and $C$ will be dense in $x_{m}=c$ and also dense in every separating boundary continuum of $E_{m}$. Thus we have the Theorem:

Theorem 1. The euclidean space $E_{m}, m \geqq 2$, is the disjoint sum of $M$, $S$ and $C$, where $M$ is of measure zero and $S+M$ and $C+M$ are each Lebesgue nonmeasurable connected sets of Van Vleck; furthermore, if $R$ is any connected region, then $R \cdot(S+M)$ and $R \cdot(C+M)$ are each Lebesgue nonmeasurable connected sets, i.e. $S+M$ and $C+M$ have these properties locally.

Proof. This follows by the methods above. By taking points of both $S$ and $C$ on every $x_{m}=c$, these $(m-1)$-planes each have these properties locally and the argument above about other separating boundary continua holds locally. Van Vleck's proof for Lebesgue nonmeasurability goes through for any intersection of $S$ and $C$ with an $(m-1)$-square and thus we have the desired local nonmeasurability; since $M$ is of measure zero, being the set of all points of $E_{m}$ with only rational coordinates, its addition does not change the property of being nonmeasurable.

Let us call the set $W$ above, i.e. the sum of the Van Vleck basic sets for all $P_{\alpha}$, the basic set of Van Vleck; let its $\left(x_{h}, x_{g}, \cdots, x_{k}\right)$ reflected sets be $W_{i}\left(i=1,2, \cdots, 2^{m}-1\right)$, each $W_{i}$ being a reflection of $W$ about some $(m-j)$-coordinate subspace $(j=1,2, \cdots, m)$, including the origin. Since being connected is invariant under reflection, if $W$ is connected each $W_{i}$ is also: in order to choose the $P_{\alpha}$ above so that $W$ is connected we need to use Zermelo's axiom of choice and the hypothesis of the continuum. To do this we may follow the well known method of Knaster and Kuratowski in [2, p. 248], well-ordering the class $\left\{B_{\gamma}\right\}$ of separating boundary continua of $E_{m}$ : however we will use the class $\left\{B_{\alpha}^{\prime}\right\}$ defined below instead, since its use will make $W$ not only connected but also locally connected. Since 
$E_{m}$ is perfectly separable it has a countable base $\left\{R_{i}\right\}$ of regions: for each $R_{i}$ and each $B_{\gamma}$ consider the nondegenerate maximal connected subsets of $R_{i} \cdot B_{\gamma}$, letting $\left\{B_{\alpha}^{\prime}\right\}$ be the set of these. Since $\left\{B_{\alpha}^{\prime}\right\}$ is of the power of the continuum we can well-order it:

$$
B_{1}^{\prime}, B_{2}^{\prime}, \cdots ; \cdots, B_{\alpha}^{\prime}, \cdots \quad \alpha<\Omega,
$$

where $\Omega$ is as above the first uncountable ordinal.

We can choose $P_{1} \in B_{1}^{\prime}-M$; then we can choose $P_{2} \in B_{2}^{\prime}$ $\cdot\left(E_{m}-M-V_{1}\right)$, since there are only a ccuntable number of points in $M+V_{1}$, these being as above. By transfinite induction we can choose $P_{\alpha} \in E_{m}-M-\left(V_{1}+V_{2}+\cdots+V_{B}+\cdots\right)$ where $V_{\beta}(\beta<\alpha)$ is already taken as above and also take $P_{\alpha} \in B_{\alpha}{ }^{\prime}$. It is possible that the sum of the Van Vleck basic sets for all $P_{\alpha}, \in B_{\alpha}^{\prime}$, we here take are not enough to give the basic set $W$ of Van Vleck above, i.e. other $P_{\boldsymbol{\alpha}}$ still need to be taken; however the sum of those for $P_{\alpha} \in B_{\alpha}^{\prime}$ is both connected and locally connected, and these properties are not destroyed by the inclusion of additional points $P_{\alpha}$. Thus it follows that both $W$ and each $W_{i}$ are connected and locally connected. Hence we have:

Theorem $1^{\prime}$. The euclidean space $E_{m}, m \geqq 2$, is the disjoint sum of sets $M, W$ and $W_{i}\left(i=1,2, \cdots, 2^{m}-1\right)$ each dense in $E_{m}$, where $M$ is of measure zero, $W$ and each $W_{i}$ are locally connected, Lebesgue nonmeasurable, connected sets and each has the nonmeasurability property (Theorem 1) locally: here $W$ is the basic set of Van Vleck and each $W_{i}$ is a reflection of $W$ about some $(m-j)$-coordinate space $(j=1,2, \cdots, m)$.

Proof. This is given above completely.

By using the hypothesis of the continuum and Zermelo's axiom we can show that there is a Van Vleck basic set $W$ which is almost, i.e. except for a set of measure zero, an indecomposable connected set $W^{\prime}$ dense in $E_{m}$. To do this we take as in [3, p. 818] a countable set $\left\{Z_{i}\right\}$ of disjoint cylinders such that, if $R$ and $R^{\prime}$ are any two disjoint regions of $E_{m}$, there exists a separating boundary continuum of $R$ contained in some $Z_{i}$ plus the boundary of $R^{\prime}$ : these cylinders cause $W^{\prime}$ to be indecomposable by a well known method used in the proof for $M$ in [3, p. 818]. Consider the class $\left\{B_{\alpha}\right\}$ of all separating boundary continua of $E_{m}$. Let, for $B_{\alpha}, B_{\alpha}^{\prime \prime}$ consist of all points of $B_{\alpha}$ not contained in an open subset of $B_{\alpha} \cdot Z_{i}$ for any $Z_{i}$. Let $B_{\alpha}^{\prime}=B_{\alpha}^{\prime \prime}$ $-U Z_{i}$. It is known [4, Theorem 44, p. 30] that $B_{\alpha}^{\prime}$ is uncountable. We can choose the $P_{\alpha}$ for $W^{\prime}$ now using these $B_{\alpha}^{\prime}$ as we used $B_{\alpha}^{\prime}$ above in the proof of 'Theorem $1^{\prime}$ : just as there we may have to take other $P_{\alpha}$ besides the $P_{\alpha} \in B_{\alpha}^{\prime}$. The set $W^{\prime}$ consists of the sum of the 
Van Vleck basic sets for all these $P_{\alpha}$, except for points that may be in $\mathrm{U} Z_{i} ; W^{\prime}$ may not be a basic set $W$ of Van Vleck but is except for $W \cdot \cup Z_{i}$. Since each $Z_{i}$ is of measure zero, $\cup Z_{i}$ is also; hence $W^{\prime}$ is almost a basic set of Van Vleck and it is Lebesgue nonmeasurable. Since each $W^{\prime} \cdot B_{\alpha}^{\prime} \neq 0, W^{\prime}$ is connected and is indecomposable by the well known type of proof used in [3, p. 818]. Since these properties are invariant under reflection, each $W_{i}^{\prime}$ has them also and we have:

TheOREM 2. The euclidean space $E_{m}, m \geqq 2$, is the disjoint sum of $M^{\prime}$, $W^{\prime}$ and $W_{i}^{\prime}\left(i=1,2, \cdots, 2^{m}-1\right)$ each dense in $E_{m}$, where $W_{i}^{\prime}$ is a reflection of $W^{\prime}$ about some $(m-j)$-coordinate subspace $(j=1,2, \cdots, m)$, $W^{\prime}$ is almost a basic set of Van Vleck, $W^{\prime}$ and each $W_{i}^{\prime}$ are Lebesgue nonmeasurable indecomposable connected sets; and $M^{\prime}$ is of measure zero.

Proof. This is given above, noting that we take $M^{\prime}=M+\cup Z_{t}$ $-U W_{i}^{\prime}$ plus the reflections of $U Z_{t}-U W_{i}^{\prime}(t=1,2, \cdots)$. The same proof would give a similar theorem where $W^{\prime}$ is $n$-indecomposable.

'Theorem 3. The euclidean plane $E_{2}$ is the sum of the sets $M^{\prime}, W^{\prime}$ and $W_{i}^{\prime}\left(i=1,2, \cdots, 2^{m}-1\right)$ each dense in $E_{2}$, where $W^{\prime}$ is almost a basic set of Van Vleck, $W_{i}^{\prime}$ is a reflection of $W^{\prime}$ about some $(m-j)$ coordinate subspace $(j=1,2, \cdots, m)$, and $W^{\prime}$ and $W_{i}^{\prime}$ are each a Lebesgue nonmeasurable biconnected set with origin $p$ as dispersion point; $M^{\prime}, W^{\prime}-p$, and $W_{i}^{\prime}-p$ are disjoint.

Proof. Let $\left\{Z_{i}\right\}$ here be the class of straight lines $x_{1}=v x_{2}$, where $v$ takes on all rational values. Let $P_{\alpha}$ now be taken exactly in relation to $\left\{Z_{i}\right\}$ as was done in the proof of Theorem 2. Let $W^{\prime}$ consist of $p$ plus the sum of the Van Vleck basic sets for the $P_{\alpha}$ as in that proof, taking $W^{\prime} \cdot \cup Z_{i}=0$. Since each $W^{\prime} \cdot B_{\alpha}^{\prime} \neq 0, W^{\prime}$ is connected as in fact is $p+\cup P_{\alpha}$. We note that $W_{i}^{\prime} \supset p$.

Suppose that $W^{\prime}-p$ contains a subarc $t$ of some $x_{1}=v^{\prime} x_{2}$, where $v^{\prime}$ is irrational. Then $W_{1}^{\prime}$ contains a reflection, $t^{\prime}$ say, of $t$. Also, except for points in $U Z_{i}$, because of the homogeneity of Van Vleck's construction $W^{\prime}$ must contain arcs parallel to $t$, at least one of which, $t^{\prime \prime}$ say, crosses $t^{\prime}$. But as $t^{\prime} \cdot \cup Z_{i}=0$ we see that $t^{\prime}$ and $t^{\prime \prime}$ must intersect in points not in $U Z_{i}$; hence this intersection is common to $W^{\prime} \cdot W_{i}^{\prime},=0$, which is a contradiction. Thus $W^{\prime}-p$ must be totally disconnected and so it follows that $W^{\prime}$, and each $W_{i}^{\prime}$, must be biconnected with $p$ as dispersion point. Therefore the theorem is true.

From the proofs above we can obtain the maximal interlacing of connected sets under Van Vleck's construction as follows. We will call $K=\bigcup P_{\alpha}$ the Van Vleck core set. We note that our proofs of 
Theorems $1^{\prime}$ and 2 give that $K$ is connected and in Theorem 3 give that $K$ is totally disconnected but $K+p$ is connected: in all of these $K \cdot \cup Z_{i}=0$. We see that (1) above gives infinitely many transformations of $K$ : these, together with the $\left(x_{h}, x_{g}, \cdots, x_{k}\right)$-reflections above, we will call Van Vleck transformations. These include rational translations when $p=0$ in (1), expansions and contractions when $u=0$, or combinations of these including expansions in some directions and contractions in others, or any of these combined with translations or combined with reflections. From basic definitions we see that the properties of having measure zero, or measure nonzero, or of being nonmeasurable are invariant under these transformations. Also they leave topological properties invariant. That the sets we get by using one of the Van Vleck transformations on $K$ is Lebesgue nonmeasurable is seen more easily by noting that except for expansion and contraction they are similar to sets of Vitali and the methods used for Vitali sets will show all of these nonmeasurable. Hence we have:

TheOREM 4. For $m \geqq 2$ and $M$ as above $E_{m}-M$ is the disjoint sum of the Van Vleck core set $K$ together with the sets $K_{i}(i=1,2, \cdots)$ where the $K_{i}$ are obtained by taking all possible Van Vleck transformations of $K: K$, and so each $K_{i}$, is Lebesgue nonmeasurable. We can take: (1) $K$, and so each $K_{i}$, a connected, locally connected set as is $W$ in Theorem $1^{\prime}$; or (2) $K$, and so each $K_{i}$, is an indecomposable connected set; or (3), for $m=2, K+p$, and so cach $K_{i}+p_{i}$, is a biconnected set, each with dispersion point $p$ or $p_{i}$ in $M$. Each one of the sets $M, K$ and $K_{i}$ is dense in $E_{m}$.

\section{REFERENCES}

1. E. B. Van Vleck, On nonmeasurable sets of points with an example, Trans. Amer. Math. Soc. vol. 9 (1908) pp. 237-244.

2. B. Knaster and C. Kuratowski, Sur les ensembles connexes, Fund. Math. vol. 2, pp. 206-253.

3. P. M. Swingle, Higher dimensional indecomposable connected sets, Proc. Amer. Math. Soc. vol. 8 (1957) pp. 816-819.

4. R. L. Moore, Foundations of point set theory, Amer. Math. Soc. Colloquium Publications vol. 13, 1932.

ThF. LiIVERsity of Miami 